\title{
Infarction in the territory of the anterior cerebral artery: clinical
} study of 5 I patients

\author{
Adrià Arboix*1,2, Luis García-Eroles ${ }^{3}$, Núria Sellarés ${ }^{1}$, Agnès Raga ${ }^{1}$, \\ Montserrat Oliveres ${ }^{1}$ and Joan Massons ${ }^{1}$
}

Address: ${ }^{2}$ Unit of Cerebrovascular Diseases, Service of Neurology, Hospital Universitari del Sagrat Cor, Universitat de Barcelona, Barcelona, Spain, ${ }^{2}$ CIBER de Enfermedades Respiratorias (CB06/06), Instituto Carlos III, Madrid, Spain and ${ }^{3}$ Unit of Organization, Planning and Information Systems, Consorci Sanitari del Maresme, Barcelona, Spain

Email: Adrià Arboix* - aarboix@hscor.com; Luis García-Eroles - lgarciaer@csdm.es; Núria Sellarés - airuncapri@hotmail.com; Agnès Raga - agnesraga@gmail.com; Montserrat Oliveres-11466moi@comb.es; Joan Massons - jmassonsc@terra.es

* Corresponding author

Published: 9 July 2009

BMC Neurology 2009, 9:30 doi:10.1186/147/-2377-9-30
Received: 30 March 2009

Accepted: 9 July 2009

This article is available from: http://www.biomedcentral.com/I47I-2377/9/30

(c) 2009 Arboix et al; licensee BioMed Central Ltd.

This is an Open Access article distributed under the terms of the Creative Commons Attribution License (http://creativecommons.org/licenses/by/2.0), which permits unrestricted use, distribution, and reproduction in any medium, provided the original work is properly cited.

\begin{abstract}
Background: Little is known about clinical features and prognosis of patients with ischaemic stroke caused by infarction in the territory of the anterior cerebral artery (ACA). This single centre, retrospective study was conducted with the following objectives: a) to describe the clinical characteristics and short-term outcome of stroke patients with ACA infarction as compared with that of patients with ischaemic stroke due to middle cerebral artery (MCA) and posterior cerebral artery (PCA) infarctions, and b) to identify predictors of ACA stroke.
\end{abstract}

Methods: Fifty-one patients with ACA stroke were included in the "Sagrat Cor Hospital of Barcelona Stroke Registry" during a period of 19 years (1986-2004). Data from stroke patients are entered in the stroke registry following a standardized protocol with $|6|$ items regarding demographics, risk factors, clinical features, laboratory and neuroimaging data, complications and outcome. The characteristics of these 5 I patients with ACA stroke were compared with those of the 1355 patients with MCA infarctions and 232 patients with PCA infarctions included in the registry.

Results: Infarctions of the ACA accounted for I.3\% of all cases of stroke $(n=3808)$ and $1.8 \%$ of cerebral infarctions $(n=2704)$. Stroke subtypes included cardioembolic infarction in $45.1 \%$ of patients, atherothrombotic infarction in $29.4 \%$, lacunar infarct in $11.8 \%$, infarct of unknown cause in $11.8 \%$ and infarction of unusual aetiology in $2 \%$. In-hospital mortality was $7.8 \%(n=4)$. Only 5 (9.8\%) patients were symptom-free at hospital discharge. Speech disturbances (odds ratio [OR] = $0.48)$ and altered consciousness $(O R=0.3 \mathrm{I})$ were independent variables of $A C A$ stroke in comparison with MCA infarction, whereas limb weakness $(O R=9.1 \mathrm{I})$, cardioembolism as stroke mechanism $(O R=2.49)$ and sensory deficit $(O R=0.35)$ were independent variables associated with ACA stroke in comparison with PCA infarction.

Conclusion: Cardioembolism is the main cause of brain infarction in the territory of the ACA. Several clinical features are more frequent in stroke patients with $A C A$ infarction than in patients with ischaemic stroke due to infarction in the MCA and PCA territories. 


\section{Background}

Cerebral infarcts in the territory of the anterior cerebral artery (ACA) are infrequent and yet few studies have specifically assessed the clinical characteristics of stroke patients with ACA infarction [1]. So far, single case reports and small series of ACA stroke have been reported and, in most cases, patients with ACA infarction are included in group of hemispheric cerebral infarction as a whole, independently of the different vascular topography of lesions [1-3]. In this respect, some aspects of the natural history of ACA infarction, such as aetiology, clinical features and prognosis have not been sufficiently documented. Moreover, the differential clinical profile between ischaemic stroke caused by infarctions in the territories of the ACA, middle cerebral artery (MCA) and posterior cerebral artery (PCA) is poorly defined, probably because separate analysis of ACA infarction as an individual clinical entity is rarely performed.

This single centre, retrospective study was conducted with the following aims: a) to describe the clinical characteristics and short-term outcome of stroke patients ACA territory infarction as compared with that of patients with ischaemic stroke due to MCA and PCA infarctions, and b) to identify predictors of ACA stroke.

\section{Methods}

The database of the "Sagrat Cor Hospital of Barcelona Stroke Registry" with data of 3808 acute stroke patients was searched for those with a diagnosis of ischaemic stroke caused by occlusion in the territory of the ACA who were admitted consecutively to the Department of Neurology of the Sagrat Cor Hospital (an acute-care 350-bed teaching hospital in the city of Barcelona) between January 1986 and December 2004. Details of this on-going hospital-based stroke registry have been previously reported [4]. Data from stroke patients are entered following a standardised protocol with 161 items regarding demographics, risk factors, clinical features, laboratory and neuroimaging data, complications and outcome.

Subtypes of stroke were classified according to the Cerebrovascular Study Group of the Spanish Neurological Society [5]. These criteria has been used by our group in previous studies $[4,6,7]$ and are consistent with those included in a recent review [8]. Definitions of cerebrovascular risk factors were those used in previous studies $[4,6,7,9]$. The distribution of patients in the database according to the different stroke subtypes were as follows: transient ischaemic attack (TIA) $(n=612)$, brain infarction $(n=2703)$, primary intracerebral haemorrhage $(n=$ 407), spontaneous subdural hematoma $(n=38)$ and spontaneous epidural hematoma $(n=1)$. Causes of ischaemic stroke were atherothrombosis in 770 cases, cardioembolism in 763, lacunes in 733 and unusual aetiologies in 114 . In 323 cases, the aetiology was unknown.
For the purpose of this study, all cases of ischaemic cerebral infarction diagnosed in 2704 patients were collected. The region of the infarction was identified on computerized tomographic (CT) scans and/or magnetic resonance imaging (MRI) studies and then topographies of the ACA $(n=51)$, MCA $(n=1355)$ and PCA $(n=232)$ were selected. These three vascular territories were defined according to previously published and validated maps of cerebral vascular territories [10] and used in other studies $[4,6,7,9]$. The objective of this clinical study was to assess differential features in aetiology, risk factors, clinical findings and early outcome between patients with ACA stroke and those with MCA and PCA infarctions. Prior to conducting the study, approval was obtained from the Ethical Committee of Clinical Research of the hospital.

All patients were admitted to the hospital within 48 hours of onset of symptoms. On admission, demographic characteristics; salient features of clinical and neurological examination and results of laboratory tests (blood cell count, biochemical profile, serum electrolytes, urinalysis); chest radiography; twelve-lead electrocardiography; and brain CT and/or MRI were recorded. Angio-MRI was obtained during hospitalisation in $33.3 \%$ of patients, echo-Doppler of the supra-aortic trunks in 60\%, and arterial digital subtraction angiography in $9.8 \%$. Other investigations included echocardiography in $56 \%$ of patients and lumbar puncture in $2 \%$. Degree of clinical disability at discharge from the hospital was evaluated according to modified Rankin scale (mRS) [11], and causes of death according to the criteria of Silver et al. [12].

\section{Statistical analysis}

Demographic characteristics, risk factors, clinical events and outcome of ACA stroke patients and those with infarctions of the MCA and PCA territories were compared using the Student's $t$-test or the Mann-Whitney $U$ test for continuous variables and the chi-square $\left(\chi^{2}\right)$ test (with Yate's correction when necessary) for categorical variables. Statistical significance was set at $P<0.05$. Variables were subjected to multivariate analysis with a logistic regression procedure and forward stepwise selection if $P<0.10$ after univariate testing. The effect of variables on the presence of infarction caused by occlusion of the ACA versus infarcts of the MCA and the PCA was studied in two multiple regression models based on demographic, vascular risk factors, and clinical and neuroimaging variables, in which the absence (codified as 0 ) or presence (codified as 1) of ACA infarction was the dependent variable. The level of significance was set as 0.15 , and the tolerance level at 0.0001 . The maximum likelihood approach was used to estimate weights of the logistic parameters. Odds ratio and $95 \%$ confidence intervals (CI) were calculated from the beta coefficients and standard errors. The hypothesis that the logistic model adequately fitted the data was tested by means of the goodness of fit $\chi^{2}$ test. The SPSS- 
Table I: Results of univariate analysis in ischaemic stroke patients with anterior cerebral artery (ACA) infarction compared with middle cerebral artery (MCA) and posterior cerebral artery (PCA)

\begin{tabular}{|c|c|c|c|c|c|}
\hline Data & Anterior cerebral artery & Middle cerebral artery & $\begin{array}{c}\text { ACA vs MCA } \\
P \text { value* }\end{array}$ & Posterior cerebral artery & $\begin{array}{c}\text { ACA vs PCA } \\
P \text { valuet }\end{array}$ \\
\hline Total patients & 51 & 1355 & & 232 & \\
\hline Males & $27(52.9)$ & $635(46.9)$ & 0.238 & $128(55.2)$ & 0.445 \\
\hline Age, years, mean (SD) & $74.4(14.7)$ & $76(11.4)$ & 0.331 & $73.9(11.9)$ & 0.801 \\
\hline Age $\geq 85$ years & II (2I.6) & $302(22.3)$ & 0.904 & $37(15.9)$ & 0.219 \\
\hline \multicolumn{6}{|l|}{ Cerebrovascular risk factors } \\
\hline Hypertension & $28(54.9)$ & $769(56.8)$ & 0.886 & $136(58.6)$ & 0.369 \\
\hline Diabetes mellitus & $15(29.4)$ & $301(22.2)$ & 0.150 & $70(30.2)$ & 0.530 \\
\hline Valve heart disease & $I(2)$ & $95(7)$ & 0.124 & $16(6.9)$ & 0.153 \\
\hline Ischaemic heart disease & $4(7.8)$ & $223(16.5)$ & 0.066 & $36(15.5)$ & 0.111 \\
\hline Atrial fibrillation & $12(23.5)$ & $461(34)$ & 0.077 & $62(26.7)$ & 0.391 \\
\hline Congestive heart dailure & $4(7.8)$ & $88(6.5)$ & 0.431 & $9(3.9)$ & 0.190 \\
\hline TIA & $4(7.8)$ & $152(11.2)$ & 0.314 & $30(12.9)$ & 0.225 \\
\hline Previous cerebral infarction & $10(19.6)$ & $225(16.6)$ & 0.342 & $4 \mid(17.7)$ & 0.440 \\
\hline Previous cerebral haemorrhage & I (2) & $10(0.7)$ & 0.335 & $4(1.7)$ & 0.633 \\
\hline Periphery arterial disease & $2(3.9)$ & $100(7.4)$ & 0.269 & $13(5.6)$ & 0.472 \\
\hline Obesity & $4(7.8)$ & $51(3.8)$ & 0.135 & II (4.7) & 0.276 \\
\hline Alcohol abuse (> $80 \mathrm{~g} /$ day) & 0 & $39(2.9)$ & 0.232 & $\mathrm{I}(0.4)$ & 0.820 \\
\hline Smoking (> 20 cigarettes/day) & $3(5.9)$ & $132(9.7)$ & 0.261 & $20(8.6)$ & 0.376 \\
\hline Dyslipemia & $9(17.6)$ & $226(16.7)$ & 0.458 & $45(19.4)$ & 0.474 \\
\hline \multicolumn{6}{|l|}{ Clinical features } \\
\hline Sudden onset & $27(52.9)$ & $722(53.3)$ & 0.537 & $108(46.6)$ & 0.251 \\
\hline Headache & 0 & $120(8.9)$ & 0.010 & $53(22.8)$ & 0.000 \\
\hline Dizziness symptoms & $2(3.9)$ & $14(1)$ & 0.112 & $7(3)$ & 0.504 \\
\hline Seizures & I (2) & $24(1.8)$ & 0.606 & 0 & 0.180 \\
\hline Nausea, vomiting & $2(3.9)$ & $62(4.6)$ & 0.587 & $17(7.3)$ & 0.299 \\
\hline Speech disturbances & $22(43.1)$ & $816(60.2)$ & 0.011 & $70(30.2)$ & 0.054 \\
\hline Altered consciousness & $4(7.8)$ & $278(20.5)$ & 0.014 & $28(12.1)$ & 0.277 \\
\hline
\end{tabular}


Table I: Results of univariate analysis in ischaemic stroke patients with anterior cerebral artery (ACA) infarction compared with middle cerebral artery (MCA) and posterior cerebral artery (PCA) (Continued)

\begin{tabular}{|c|c|c|c|c|c|}
\hline Motor deficit ${ }^{\ddagger}$ & $44(86.3)$ & $1145(84.5)$ & 0.459 & $90(38.8)$ & 0.000 \\
\hline Sensory & $15(29.4)$ & $554(40.9)$ & 0.066 & $119(51.3)$ & 0.003 \\
\hline Aetiological stroke subtypes & & & 0.337 & & 0.001 \\
\hline Atherothrombotic & $15(29.4)$ & $395(29.2)$ & & $68(29.3)$ & \\
\hline Lacunar & $6(11.8)$ & $301(22.2)$ & & $80(34.5)$ & \\
\hline Cardioembolic & $23(45.1)$ & $482(35.6)$ & & $50(21.6)$ & \\
\hline Unknown aetiology & $6(11.8)$ & $124(9.2)$ & & $20(8.6)$ & \\
\hline Unusual cause & $I(2)$ & $52(3.9)$ & & $14(6)$ & \\
\hline Symptom-free at discharge & $5(9.8)$ & $149(11)$ & 0.507 & $45(19.4)$ & 0.072 \\
\hline Cardiac events & $2(3.9)$ & $78(5.8)$ & 0.436 & $6(2.6)$ & 0.439 \\
\hline Infectious events & $6(11.8)$ & $237(17.5)$ & 0.194 & $15(6.5)$ & 0.155 \\
\hline Respiratory events & $4(7.8)$ & $176(13)$ & 0.196 & $12(5.2)$ & 0.321 \\
\hline $\begin{array}{l}\text { Length of hospital stay, median } \\
\text { (25th-75th percentile) }\end{array}$ & $12(10-24)$ & $13(9-23)$ & 0.895 & $12.8(8-22)$ & 0.601 \\
\hline In-hospital deaths & $4(7.8)$ & $235(17.3)$ & 0.048 & $9(3.9)$ & 0.190 \\
\hline
\end{tabular}

Data are $n(\%)$ unless otherwise stated.

$* P=0.337 ;+P=0.001$.

$\dagger$ Association with motor hemonegligence in $11.8 \%$; concomitant presence of grasping in $17.6 \%$; callosal disconnection syndrome in $5.9 \%$.

$\mathrm{PC}+$ and the BMDP computer programmes were used for statistical analyses.

\section{Results}

The 51 patients with ischaemic stroke caused by infarction of the ACA territory accounted for $1.3 \%$ of all cases of stroke $(n=3420)$ and $1.8 \%$ of cerebral infarction $(n=$ 2407 ) included in the stroke registry. There were 27 men and 24 women with a mean (SD) age of 71.4 (14.7) years. Eleven patients aged 85 years or older. The following vascular risk factors in a decreasing order of frequency were observed: hypertension (54.9\%), diabetes mellitus $(29.4 \%)$, atrial fibrillation $(23.5 \%)$ and dyslipemia $(17.6 \%)$. History of previous TIA was present in $7.8 \%$ of patients. Sudden onset of neurological deficit was recorded in $96.3 \%$ of cases. Speech disturbances (dysarthria, aphasia) occurred in $43.1 \%$ of cases, sensory deficit in $29.4 \%$ and decreased level of consciousness in $7.8 \%$. Stroke subtypes included cardioembolic infarction in $45.1 \%$ of patients, atherothrombotic infarction in $29.4 \%$, lacunar infarct in $11.8 \%$, infarct of unknown cause in $11.8 \%$ and infarction of unusual aetiology in $2 \%$.
Four patients died during the hospital stay, with an inhospital mortality rate of $7.8 \%$. Causes of death included herniation of the brain in 1 , sudden death in 1 , pneumonia in 1 and septicaemia in 1 . The median length of hospitalization was 12 days (25th-75th percentile, 10-24 days). Only $5(9.8 \%)$ patients were symptom-free at the time of hospital discharge (mRS grade 0 ). Of the remaining 42 patients, 20 had moderate disability (mRS grade 3), 17 moderately severe disability (mRS grade 4 ) and 5 severe disability ( $\mathrm{mRS}$ grade 5 ).

As shown in Table 1, ischaemic heart disease, atrial fibrillation, headache at the time of stroke onset, speech disturbances, altered consciousness and sensory deficit were less frequent in stroke patients with ACA infarction than in those with MCA infarction. On the other hand, headache, sensory deficit and symptom-free at discharge were less frequent, and motor deficit, speech disturbances and cardioembolism as the aetiology of stroke more frequent in patients with ACA infarction than in those with PCA infarction (Table 1). 
Table 2: Variables associated with ischaemic stroke caused by anterior cerebral artery (ACA) infarction

\begin{tabular}{|c|c|c|c|c|}
\hline Variable & $\beta$ & SE $(\beta)$ & $\begin{array}{l}\text { Odds ratio } \\
(95 \% \mathrm{Cl})\end{array}$ & $p$ \\
\hline \multicolumn{5}{|l|}{$A C A$ versus $M C A$ infarctions } \\
\hline \multicolumn{5}{|c|}{ Model based on demographics, vascular risk factors and clinical variables* } \\
\hline Speech disturbances (dysarthria, aphasia) & -0.728 & 0.289 & $0.48(0.27-0.85)$ & 0.012 \\
\hline Altered consciousness & -1.159 & 0.526 & $0.31(0.11-0.88)$ & 0.028 \\
\hline
\end{tabular}

ACA versus PCA infarctions

Model based on demographics, vascular risk factors, clinical features and topographic and aetiological variablest

\begin{tabular}{|c|c|c|c|c|}
\hline Motor deficit & 2.210 & 0.445 & $9.11(3.8-21.8)$ & 0.000 \\
\hline Cardioembolism & 0.914 & 0.269 & $2.49(1.21-5.14)$ & 0.013 \\
\hline Sensory deficit & -1.036 & 0.377 & $0.35(0.17-0.74)$ & 0.006 \\
\hline
\end{tabular}

In the multivariate analysis (Table 2), speech disturbances (odds ratio $[\mathrm{OR}]=0.48,95 \% \mathrm{CI} 0.27-0.85$ ) and altered consciousness $(\mathrm{OR}=0.31,95 \% \mathrm{CI} 0.11-0.88)$ were independent variables of ACA stroke in comparison with MCA infarction, whereas motor deficit $(\mathrm{OR}=9.11,95 \% \mathrm{CI} 3.8-$ 21.8) cardioembolism as stroke mechanism $(\mathrm{OR}=2.49$, 95\% CI 1.21-5.14) and sensory deficit (OR $=0.35,95 \%$ CI 0.17-074) were independent variables associated with ACA stroke as compared with PCA infarction.

\section{Discussion}

Data regarding the frequency of cerebral infarctions in the territory of the ACA in the different stroke registries are scarce, the number of which varies between 27 in the Lausanne Stroke Registry [13] and 47 in the Ege Stroke Registry [14]. The clinical series of 51 patients here described is the largest group so far reported in the literature (Table 3 ). The present results indicate that infarctions in the ACA territory is a subgroup of unusual cerebral infarcts, accounting for $1.8 \%$ of all cases of stroke and $1.3 \%$ of all cases of cerebral infarctions. This percentage is similar to $1.9 \%$ observed in the series of Moulin et al. [15], slightly higher than $1.1 \%$ in the series of Vemmos et al. [16] and 1.3\% of Kumral et al. [13] but lower than 2.3\% in the clinical series of Gandehari and Izadi [17].

It should be noted that $21.6 \%$ of our patients were older than 85 years of age. This finding is consisted with results of other studies [18-20] and highlights the increasing prevalence and clinical relevance of first-ever stroke in the oldest old population segment of developed countries.

The aetiology of ACA infarctions is poorly defined, although in our study, the most frequent aetiopathogenic mechanism was cardioembolism in $45 \%$ of cases, followed by atherothrombosis in $29 \%$ and lacunes in $12 \%$. In $12 \%$ of the cases, the cause was unknown. Infarctions were considered of cardioembolic origin according to criteria recommended by the Cerebrovascular Study Group of the Spanish Society of Neurology [5] and defined as a medium-to-large size cerebral infarction, usually of cortical topography in which in the absence of other etiologies, some of the following emboligenous cardiac disorders are documented: atrial flutter or atrial fibrillation, intracardiac thrombus or tumor, rheumatic valve disease, mitral or aortic valve prosthesis, endocarditis, sinus node disease, left ventricular aneurysm after acute myocardial infarction, acute myocardial infarction in the acute phase (less than 3 months) or global cardiac hypokinesia. The identification of cardioembolism as the aetiology of stroke in $45 \%$ of our patients is similar to the percentages of $45.7 \%$ reported in the studies of Moulin et al. [15] and Vemmos et al. [50\%] but clearly higher than $25 \%$ found in the studies of Bougousslavki and Regli [13] and 27\% in the study of Kumral et al. [14]. However, all these percentages are consistently higher than the frequency of about 
Table 3: Cerebral infarcts in the territory of the anterior cerebral artery (ACA). Main series reported in the literature

\begin{tabular}{|c|c|c|c|c|c|c|}
\hline $\begin{array}{l}\text { First author, year } \\
\text { [reference] }\end{array}$ & Patients & Clinical series & $\begin{array}{c}\text { Frequency } \\
\text { cardioembolic } \\
\text { aetiology } \\
\text { (\%) }\end{array}$ & $\begin{array}{l}\text { Frequency total } \\
\text { infarctions } \\
(\%)\end{array}$ & $\begin{array}{c}\text { Frequency total } \\
\text { Stroke } \\
(\%)\end{array}$ & $\begin{array}{l}\text { In-hospital mortality } \\
\text { (\%) }\end{array}$ \\
\hline $\begin{array}{l}\text { Bogousslavski, } 1990 \\
\text { [13] }\end{array}$ & 27 & $\begin{array}{c}\text { Lausanne Stroke } \\
\text { Registry } \\
(n=1490)\end{array}$ & 26 & Not stated & 1.8 & Not stated \\
\hline Moulin, 2000 [15] & 35 & $\begin{array}{c}\text { Besançon Stroke } \\
\text { Registry } \\
(n=1776)\end{array}$ & 45.7 & 1.9 & Not stated & 14.3 \\
\hline Vemmos, 2000 [16] & 10 & $\begin{array}{c}\text { Athens Stroke } \\
\text { Registry } \\
(n=1042)\end{array}$ & 50 & 1.1 & 0.9 & Not stated \\
\hline Kumral, 2002 [14] & 48 & $\begin{array}{l}\text { Ege Stroke Registry } \\
\qquad(n=4750)\end{array}$ & 27 & 1.3 & I & 0 \\
\hline $\begin{array}{l}\text { Gandehari, } 2007 \\
{[17]}\end{array}$ & 32 & $\begin{array}{c}\text { Khorasan Stroke } \\
\text { Registry } \\
(n=1392)\end{array}$ & Not stated & 2.3 & Not stated & Not stated \\
\hline Present series, 2009 & 51 & $\begin{array}{l}\text { Hospital Sagrat Cor } \\
\text { Stroke Registry } \\
(n=3808)\end{array}$ & 45.1 & 1.8 & 1.3 & 7.8 \\
\hline
\end{tabular}

$20 \%$ attributed to cardioembolism when cerebral infarctions are analysed as a whole [21,22]. In this respect, this aetiological aspect is important because a diagnosis of cardioembolism has practical implications in the management of these patients. In the presence of a cerebral infarction in the ACA territory, the cardioembolic mechanism should be considered and, therefore, the possibility to indicate early anticoagulation at therapeutic doses as a secondary prevention of cardioembolic stroke [8].

Patients with infarcts in the ACA territory have a relatively favourable short-term prognosis as shown by an in-hospital mortality rate of $7.8 \%$, clearly lower that $17.3 \%$ of MCA infarctions. Other authors have reported mortality rates between $0 \%$ in the series of Kumral et al. [14] and $14.3 \%$ in the study of Moulin et al. [15].

Neuroimaging data (brain CT and/or MRI) was indispensable to confirm the vascular topography of the lesion and to classify patients into the three groups of infarcts in the territory of the ACA, MCA and PCA. The remaining diagnostic studies (laboratory data, echo-Doppler of the supra-aortic trunks, arterial digital subtraction angiography, etc.) were useful to classify patients into the different ischemic stroke subtypes.

It should be noted that ACA infarcts present a clinical profile clearly different than the remaining cerebral hemi- spheric infarctions. Speech disturbances and altered consciousness were independent variables associated with ACA infarctions as compared with MCA stroke. The lower frequency of dysarthria or aphasia is explained because both Broca's motor speech area and sensory speech area of Wernicke are located in the vascular territory of the MCA $[1,10]$. The lower frequency of decreased consciousness may be related to the small diameter of the lesion in ACA in comparison with MCA infarctions. A larger volume of brain ischaemia increases the risk of intracranial hypertension and consequently more frequent impairment of the level of vigilance at the beginning of the focal neurological deficit [1].

Variables associated with ACA stroke versus infarctions in the vascular territory of the PCA were motor deficit, cardioembolism and sensory deficit. Motor deficit with a characteristic crural distribution is the most common neurological sign of ACA stroke and was present in $86.3 \%$ of our patients as compared with a prevalence of $93.3 \%$ in the series of Kumral et al. [14] and 96\% in the series of the Lausanne Stroke Registry [13]. Infarctions in the ACA territory usually involve the paracentral component of the frontal lobe affecting motor neurons with a somotatotopic distribution mostly related to the lower extremities [1]. Cardioembolism is the most frequent aetiology of ACA infarction as compared with infarcts in the PCA territory in which cardioembolism was the third cause after 
lacunar infarctions and atherothrombosis. On the other hand, the lower frequency of sensory deficit in ACA stroke than in PCA infarction is explained because the deep vascular territory of the PCA includes the ventroposterolateral thalamic nucleus [23].

\section{Conclusion}

Cerebral infarcts in the ACA territory are infrequent and account for only $1.3 \%$ of all cases of cerebral infarction and $1.3 \%$ of all cases of stroke. Cardioembolism is the main aetiology of ACA stroke. Patients with ACA infraction have a favourable short-term prognosis and show a clinical profile different than the remaining cerebral hemispheric infarcts.

\section{Abbreviations}

ACA: anterior cerebral artery; CI: Confidence interval; CT: Computed tomography; MCA: middle cerebral artery; MRI: Magnetic resonance imaging; OR: Odds ratio; PCA: Posterior cerebral artery; ROC: Receiver operating characteristics; SD: standard deviation; TIA: Transient ischaemic attack.

\section{Competing interests}

The authors declare that they have no competing interests.

\section{Authors' contributions}

AA was the principal investigator, designed the study, diagnosed and took care of the patients, contributed to analyze the data, interpreted the results, wrote the paper, and prepared the final draft. He was also responsible for editorial decisions including the selection of the journal. LG-E was the statistician, participated in the study design, analysis and interpretation of data, and wrote the part of the paper related to the statistical analysis. NS, AR, MO and JM participated in the collection of data medical care of the patients, analysis of results, and review of the manuscript for intellectual content. All authors read and approved the final manuscript.

\section{Acknowledgements}

The study was supported by a grant from FIS PI08I5I4, Madrid, Spain. We thank Drs Miquel Balcells, Cecília Targa, Emili Comes, Ramón Pujades, Núria Anguera, and Roser Casañas for the care of many of the patients included in the study, and Dr Marta Pulido for editing the manuscript and editorial assistance.

\section{References}

I. Brust JCM, Chamorro A: Anterior cerebral artery disease. In Stroke. Pathophysiology, Diagnosis, and Management Edited by: Mohr JP, Choi DW, Grotta JC, Weir B, Wolf PhA. Philadelphia: Churchill Livingstone; 2004:101-122.

2. Grau AJ, Weimar C, Buggle F, Heinrich A, Foertler M, Neumaier S, Glahn J, Brandt T, Hacke W, Diener HC: Risk factors, outcome, and treatment in subtypes of ischemic stroke: The German Stroke Data Bank. Stroke 200I, 32:2559-2599.

3. Lee B, Hwang S, Jung S, Yu K, Lee J, Cho S, Lee SM, Song HK: The Hallym Stroke Registry: a web-based stroke data bank with an analysis of 1,654 consecutive patients with acute stroke. Eur Neurol 2005, 54:8I-87.

4. Arboix A, Morcillo C, García-Eroles L, Massons J, Oliveres M, Targa C: Different vascular risk factor profiles in ischemic stroke subtypes. The Sagrat Cor Hospital of Barcelona Stroke Registry. Acta Neurol Scand 2000, 102:264-270.

5. Arboix A, Alvarez-Sabín J, Soler L, for the Cerebrovascular Study Group of the Spanish Society of Neurology: Nomenclatura de las enfermedades vasculares cerebrales. Neurologia 1998, I3(Suppl I): I- I0.

6. Pujadas R, Arboix A, Anguera N, Oliveres M, Massons J, Comes E: Role of complex aortic atheroma plaques in the recurrence of unexplained cerebral infarction. Rev Esp Cardiol 2005, 58:34-40.

7. Marrugat J, Arboix A, García-Eroles L, Salas T, Vila J, Castell C Tresserras $R$, Elosua $R$ : The estimated incidence and case fatality rate of ischemic and hemorrhagic cerebrovascular disease in 2002 in Catalonia. Rev Esp Cardiol 2007, 60:573-580.

8. Ustrell-Roig X, Serena-Leal J: Stroke. Diagnosis and therapeutic management of cerebrovascular disease. Rev Esp Cardiol 2007, 60:753-769.

9. Arboix A, García-Eroles L, Comes E, Oliveres M, Balcells M, Pacheco $G$, Targe C: Predicting spontaneous early neurological recovery after ischemic stroke. Eur J Neurol 2003, 10:429-435.

10. Brust JMC, Sawada T, Kazui S: Anterior cerebral artery. In Stroke Syndromes Edited by: Bogousslavski J, Caplan LR. Cambridge University Press: Cambridge; 2001:439-350.

1I. Bamford JM, Sandercock PAG, Warlow CP, Slattery J: Interobserver agreement for the assessment of handicap in stroke patients. Stroke 1989, 20:828.

12. Silver FL, Norris JW, Lewis AJ, Hachinski VC: Early mortality following stroke: a prospective review. Stroke 1984, 15:492-496.

13. Bogousslavsky J, Regli F: Anterior cerebral artery territory infarction in the Lausanne Stroke Registry. Clinical and etiologic patterns. Arch Neurol 1990, 47:144-150.

14. Kumral E, Bayulkem G, Evyapan D, Yunten N: Spectrum of anterior cerebral artery territory infarction: clinical and MRI findings. Eur J Neurol 2002, 9:615-624.

15. Moulin T, Tatu L, Vuillier F, Berger E, Chavot D, Rumbach L: Role of a Stroke Data Bank in evaluating cerebral infarction subtypes: patterns and outcome of $I, 1776$ consecutive patients from the Besançon Stroke Registry. Cerebrovasc Dis 2000, I0:26I-27|

16. Vemmos KN, Takis CE, Georgilis K, Zakopoulos NA, Lekakis JP, Papamichael CM, Zis VP, Stamatelopoulos S: The Athens stroke registry: results of a five-year hospital-based study. Cerebrovasc Dis 2000, 10:133-14|

17. Ghandehari K, Izadi Z: The Khorasan Stroke Registry: results of five-year hospital-based study. Cerebrovasc Dis 2007, 23: I32-139.

18. Arboix A: Increasing relevance of acute cerebrovascular disease in very old patients. Eur J Neurol 2007, 1 4:833-834.

19. Rojas JI, Zurrú MC, Romano M, Patrucco L, Cristiano E: Acute ischemic stroke and transient ischemic attack in the very old. Risk factors and stroke subtype between patients older than 80 years and patients aged less than 80 years. Eur J Neurol 2007, 14:895-899.

20. Arboix A, García-Eroles L, Massons J, Oliveres M, Targa C: Acute stroke in very old people: clinical features and predictors of in-hospital mortality. J Am Geriatr Soc 2000, 48:36-4I.

21. Pujadas R, Arboix A, Casañas-Muñoz R, Anguera-Ferrando N: Specific cardiac disorders in 402 consecutive patients with ischaemic cardioembolic stroke. Int J Cardiol 2004, 95: I29-I34.

22. Hart RG: Cardiogenic embolism to the brain. Lancet 1992, 339:589-594.

23. Fisher CM: Thalamic pure sensory stroke: a pathologic study. Neurology 1978, 28: | |4|-| | 44 .

\section{Pre-publication history}

The pre-publication history for this paper can be accessed here:

http://www.biomedcentral.com/1471-2377/9/30/prepub 